$\mathrm{VBP}: \mathrm{AK}$

$\mathrm{XII}-3$
U. S. DEPARTMENT CF COMARCE

NATICNAI BUREATU CF STAITDARDS

Washington

Revised to November 20, 1940
Letter

Circular

IC-619

(Superseding

I. -570 )

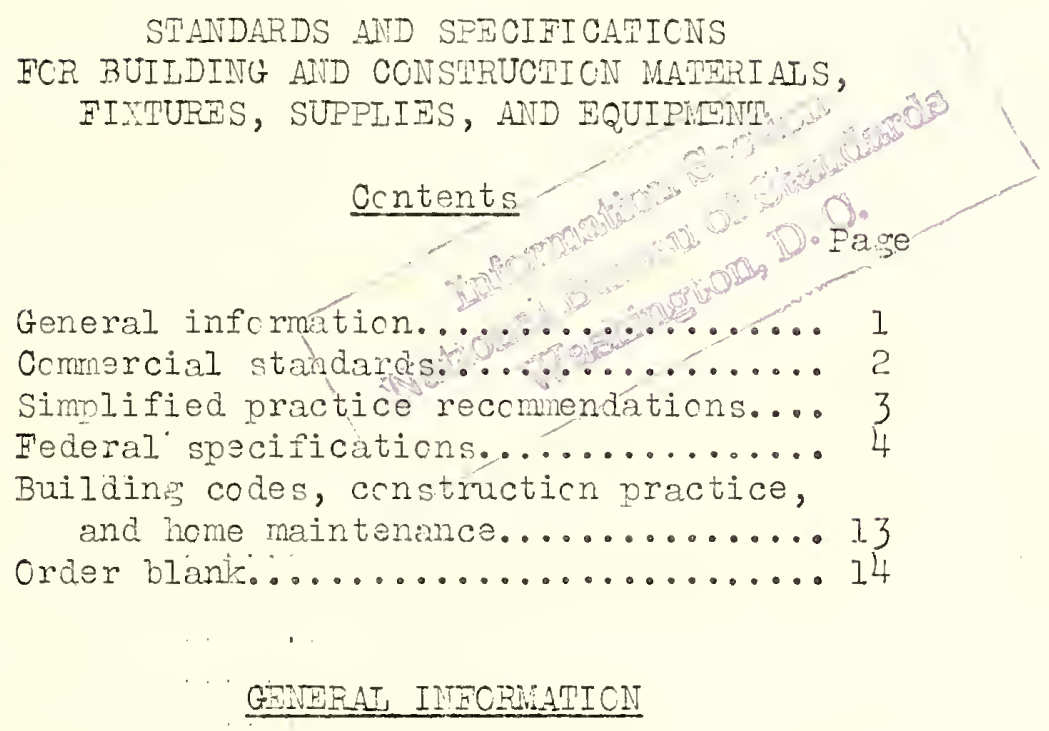

Commercial Standards, Simplified Practice Recormendations, Federal Specifications, and Bureau publications for building and construction materials, fixtures, supplies, and equipment are listed herein; and where the price is stated the publication can be cbtained from the superintendent of Documents, Gov ernnent Printing Cffice, Tashington, D. C. Federal Specifications are priced at 5 cents per ccpy except where otherwise indicated. The prices quoted are for delivery tc addrasses in the United States and its territories and possessions and in certain forein countries which extend the franking privilege. In the case of all other countries, one-third the ccst of the publication should be added to cover postage. Remittances should be made either by coumons (obtainable from the Superintendent of Dccument's in sets of 20 for $\$ 1.00$ and gcod until used), or by check or money order payable to the "Superintendent of Documents, Gcvemment Printing officell and sent to him with order.

Lists of cources of supply of materials graranteed to comply with the specification requirements have been compiled. fcr most of the cormercial Standards and Federal Specificaticas. They are indicated by an asterisk, and may be obtained free of charge from the Division of Codes and Specifications, National Bureau of Standard's, Washington, D. C.

An index of all current Federal specifications, indicating the status of the specifications and showing the price of printed copies, has been published as Section IV, Part I cf the Federal Standard Stcck Catalcg. Copies of this index may be purchased from the Superintendent of Documents, at 15 cents per copy. 
Publicaticns marked "Op" are cut of print but, in seneral, may be ccnsulted at tecinical lioraries.

Series letters with serial numbers are used to designato Bureau publications:

$B H=$ "Building and Housing" publication.

BMS = "Building Materials and Structures" publication.

C = "Circular."
$C S=$ "Commercial Standard."

$I C=$ "Letter Circular."

$W=$ "Miscellaneous Publicaticn."

$\mathrm{R}=$ "Simplified Practice Recommendation."

\section{COMNERCIAI STANDARDS}

Title

Series

Price

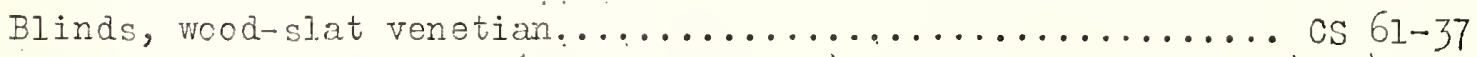

Board, fiber insulating (socond edition).............. Cs $42-35$

Burners, automatic mechanical

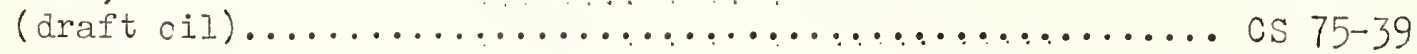

Burners, domestic, for Pennsylvania anthracite

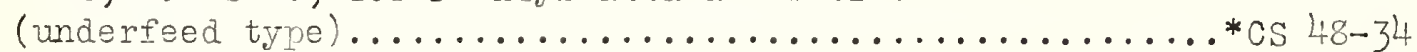

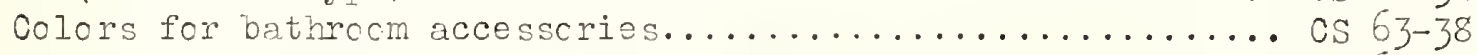

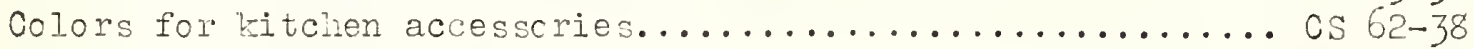

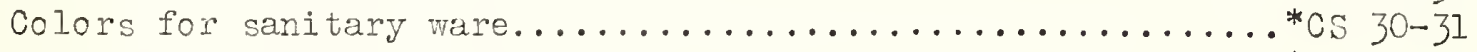

Colors and finishas for cast stcne....................... 53-35

Commercial standards and thoir value to ousiness

(second editicn)...................................... $0-40$

Dcors; old srowth Dcuslas fir standard stock

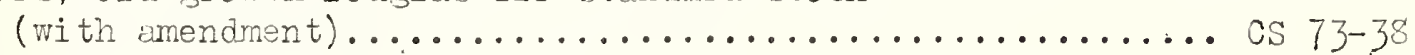

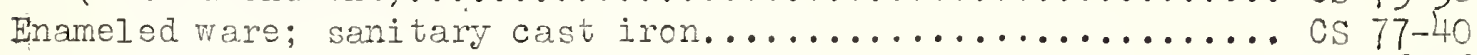

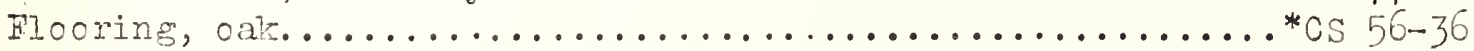

Hardware, builders' (nontemplate).............................. 22-40

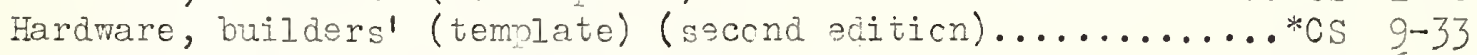

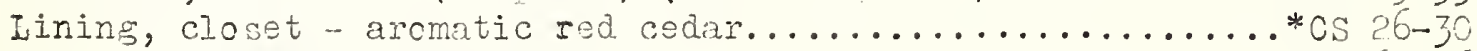

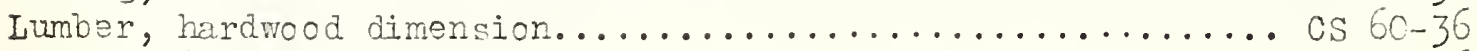

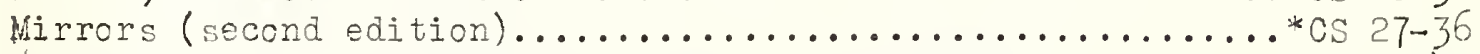

Nipples, pipe; brass, copper, steel, and wrcught iron....... CS 5-40

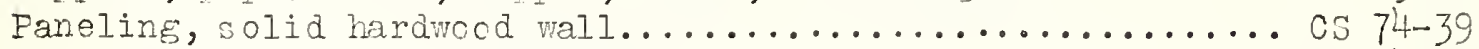

Plumbing fixtures, staple porcelain (all-clay).............. 4-29

Plumbing fixtures, staple vitrecus china (second editicn)...*CS 20-36

Plywocd, Douglas fir (domestic grades) (fcurth edition).....*CS 45-40

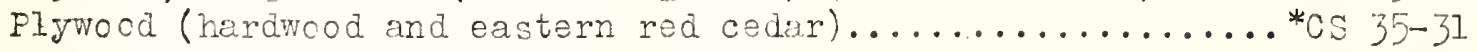

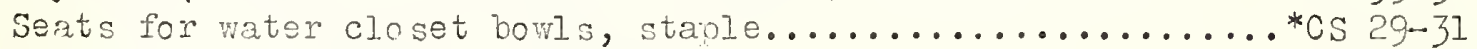

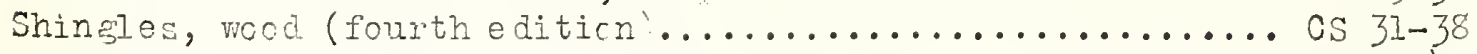

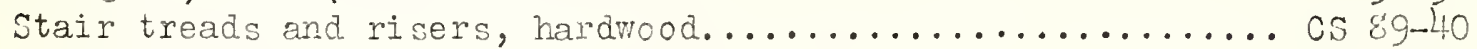

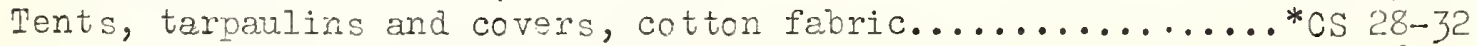

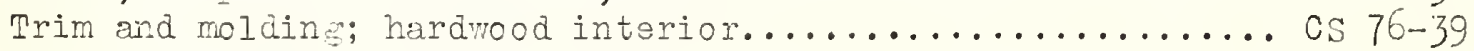

Unions, standard weight malleable iron or sieel screwed.....*CS 7-29

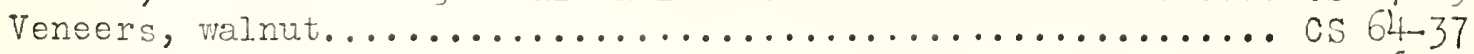

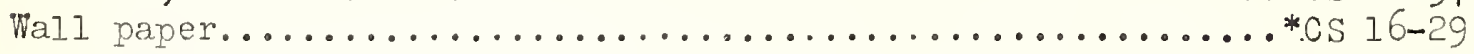

While the supply lasts, mimeosraphed copies of the above commercial Standards a re available without charpe from the Division of Trade Standards, National Bureau of Standards, Washington, D. C. 
Aggregates, coarse (crushed stone, gravel and slag)........... 163-39

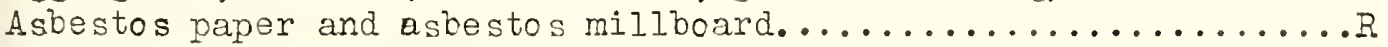

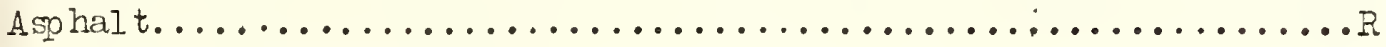

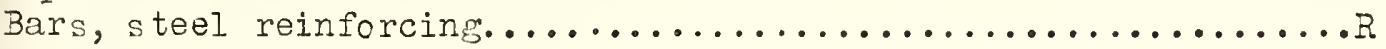

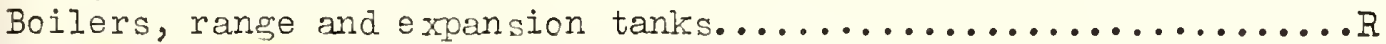

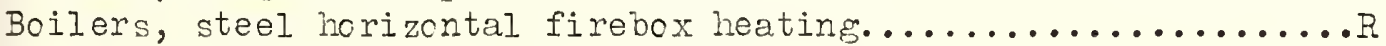

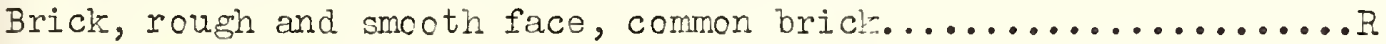

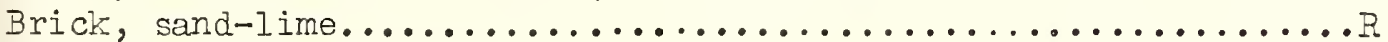

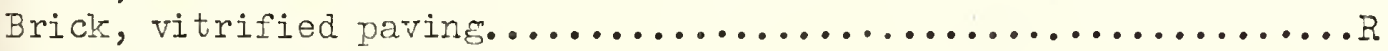

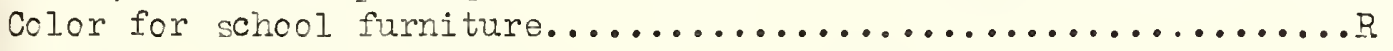

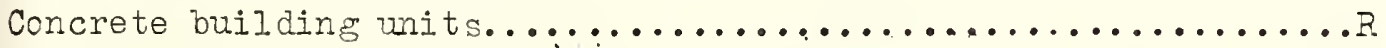
Doors, hollow motal single acting swing, frames and trim.........

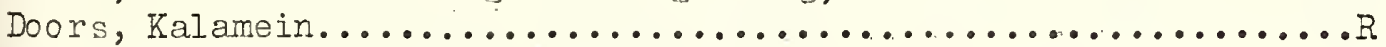
Eaves trough and conductor pipe, fittings, and ridge rolls......R

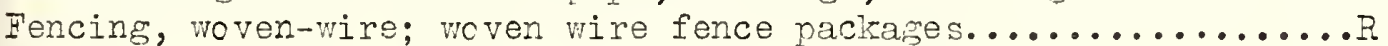

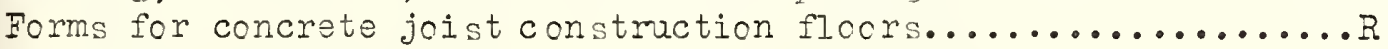

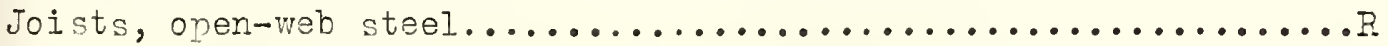

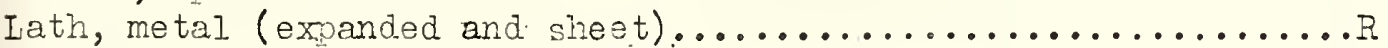

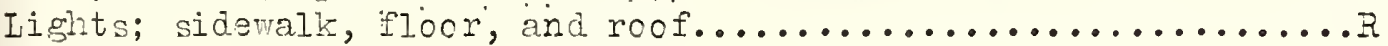

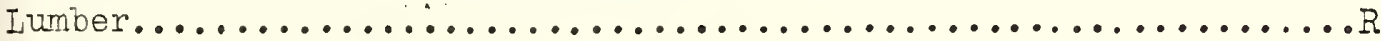

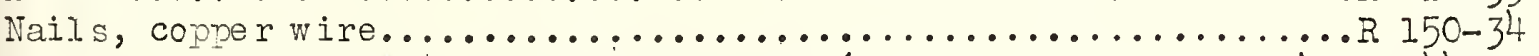
Paints, varnishes, and rolated products (shades and containers)..R $144-37$ Partitions for toilets and showers, metal.................. 101-40 Pipe, valves and fittings, wrought-iror and wrought-steel....... 57-32

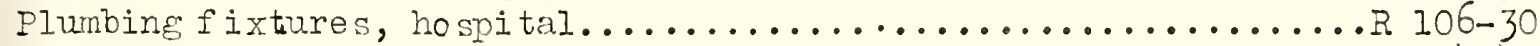

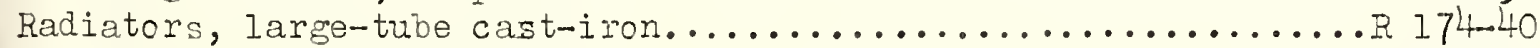

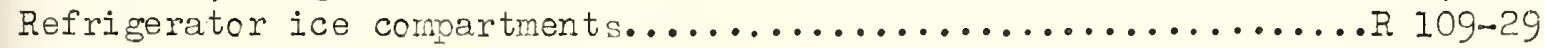

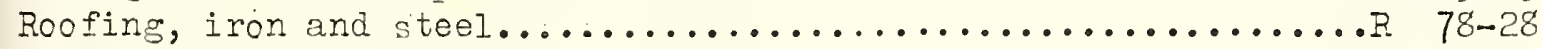

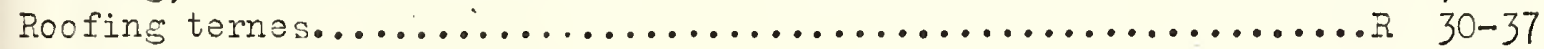

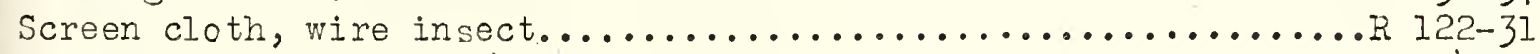

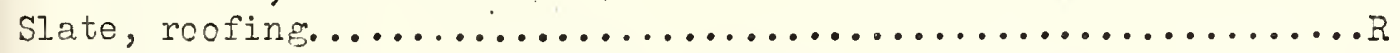

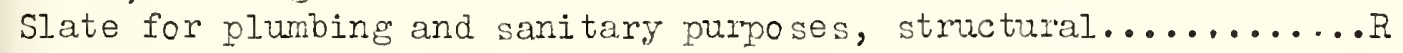

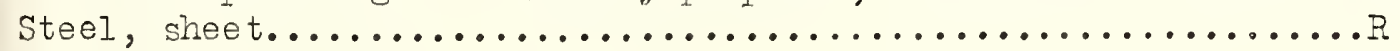

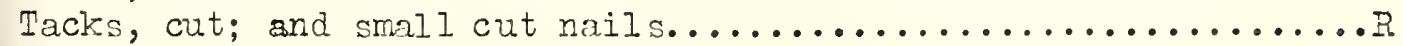

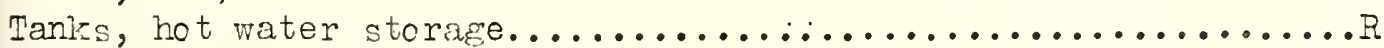

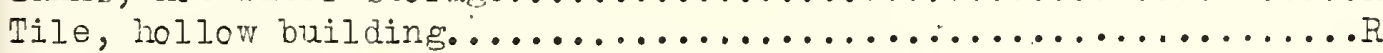

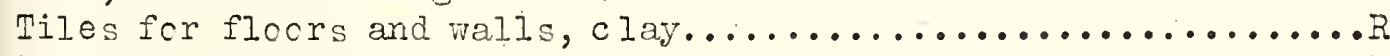

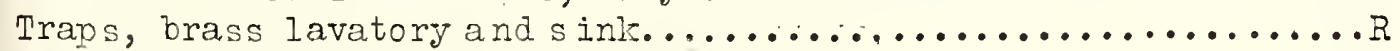

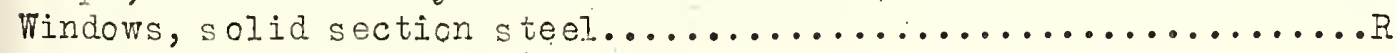
$14-28$ $13-28$

Simplified practice - Its purpose and applicaticn............ 590

Where no price is shown, publications are available in mimeographed form only from the Division of Simplified Practice, National Bureau of Standards, Washington, D. C. 
Index of Federal Specifications for Building and Construction Materials, Fixtures, Supplies, and Equipment arranged by

Commodity Groups

* Iist of Willing-to-Certify Sources of Supply has boen compiled.

Specification

symbo]

\section{ANIMAL PRODUCTS}

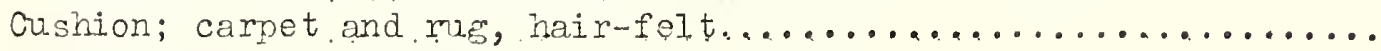

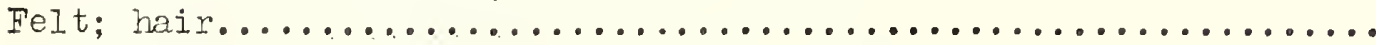
$C-C-811$

Glue; animal (for). woodworlsing.

* C-F-201a

$* \mathrm{C}-\mathrm{G}-451$

\section{CABIE AND WIRE}

Cable (armored and lead-covered-armored) and Cord (armored);

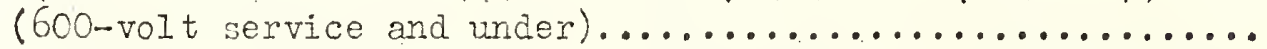

Cable and Wire; mbber-insulated, building-type, code-grade......

Cable and Wire; rubber-insulated, building-type, heat-resistant

grade (0 to 5,000-volt service).

Cable and Wire; rubber-insulated (for) other than building-

purposes, superaging-grade ( $Q$ to $8,000-v o l t$ service).

$* \mathrm{~J}-\mathrm{C}-71$

$* J-C-101 b$

*J-C-106a

*J-C-I2I

\section{CEEMICAIS}

Fire-Extinguishers; chemical, hand, carbon-tetrachloride type..... Fire-Extinguishers; chemical, hand, soda-and-acid type..........

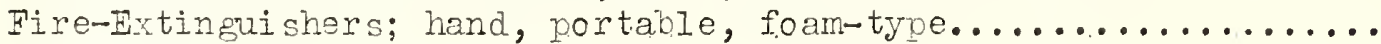
Fire-Extinguishing-Iiquid; carbon-tetrachloride base...........

$* 0-8-351$

* $0-3-355$ a

$0-F 361$

*O- $1-380$

\section{COAI-TAR. AND PRODUCTS}

Pitch; coal-tar (for) mineral-surfaced builtwup rocfing,

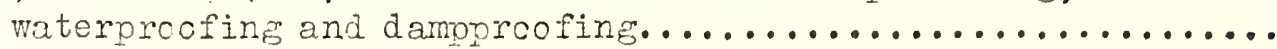

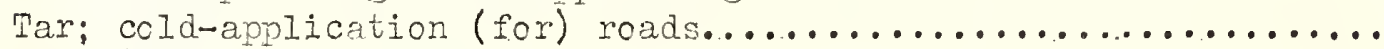

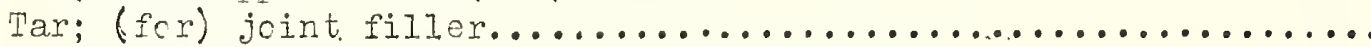

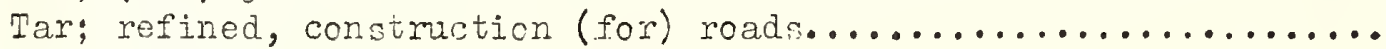

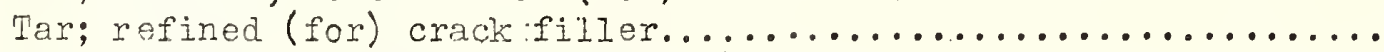

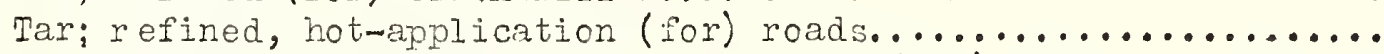

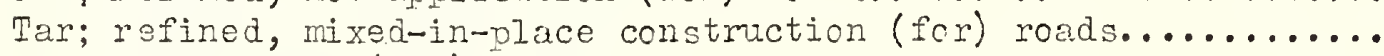

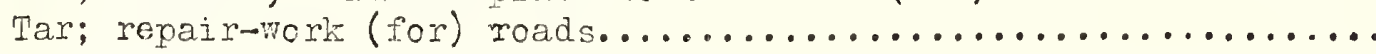

$* \mathrm{R}-\mathrm{P}-38 \mathrm{I}$

*R-T-101

* R-T-III

*R-I-I2I

*R-T-126

$* R-T-131$

$R-T-136$

$* R-T-141$

\section{CORDAGE, TWINE, AND PRODUCTS}

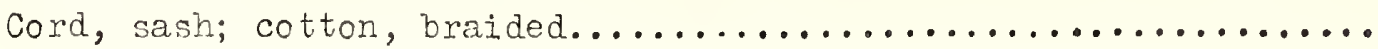


Symbol

EIECTRIC-APPAPATUS

Bases; cut-out (for) enclosed-cartriage, open-link, and

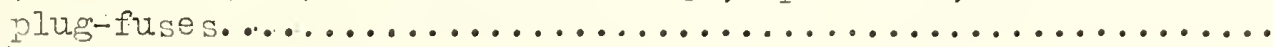

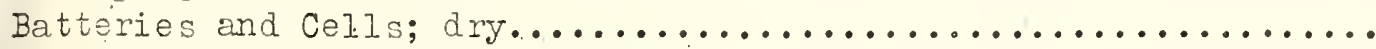
Boxes and Cutlet-Fittings, floor; (for) rigid-steel-conduit

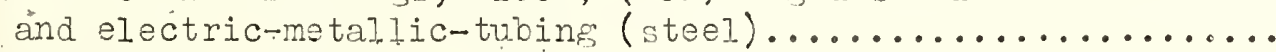

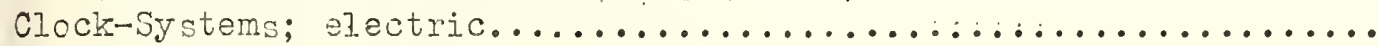
Fans, electric; bracket an d desl types, risid-blades

(for shore use).

$* w-3-81 a$

$* w-B-101 a$

WT- B-6].6

$\mathrm{W}-\mathrm{C}-471 \mathrm{a}$

W-F-101a

Fire-Alarm Systems; electric, hand-operated, positive,

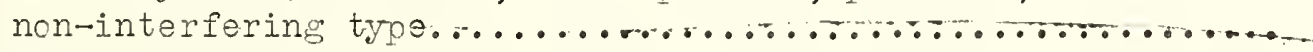

Fire-Alarm Systems; electric, hard-operated, shunt-type...........

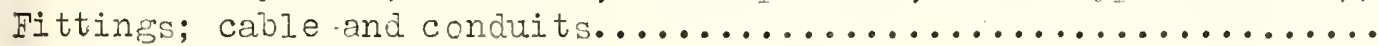
Fuses; cartridge, inclosed, nonrenewable.

Fuses; cartridge, inclosed, renewable (fusible links not separately inclo sed).

Fuses; cartridge, inclosed, renewable (fusible linlss

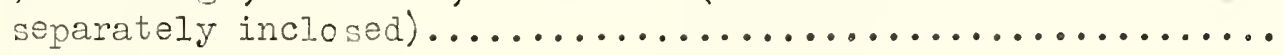

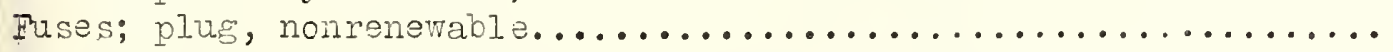
Lamps; electric, incandescent, large, tungsten-filament......... Outlet-Bodies; iron (cast or malleable), cadmium or zinc-coated. with covers and accessories (for shore use). W- $T-391$ Ti- F-396 Vi- +406 iT- -791

$* 7-7-803$

$* T-\mathrm{T}-805$

$* W-7-831$

*W-I-101d

$W-0-806$

outlet-Boxes; steel, cadmium or zinc-coated, with covers

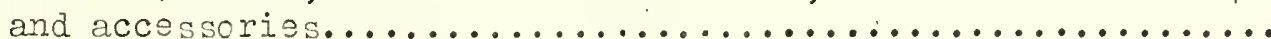

Panelboards; equipped with automatic-circuit breakers............

Panelboards; equipped with fuse-connections or switches

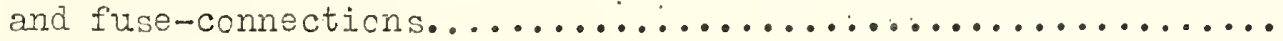

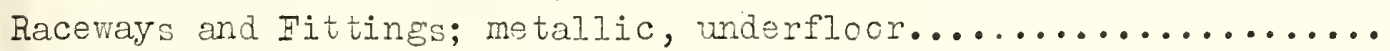

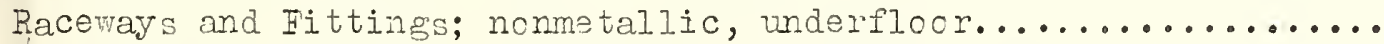
Receptacles (convenience-outlets); attachment-pluss,

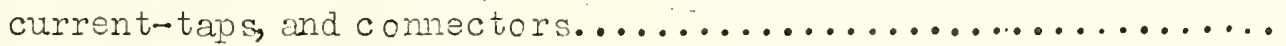
Switches; lmife, open-type, front-and rear-connected............ Switches; snap, maltiple-type and combination-devices,

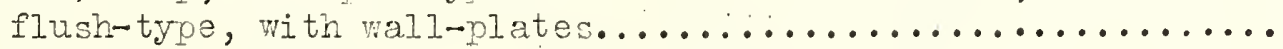
Switches; snap, single-unit, interchangeable, flush-type,

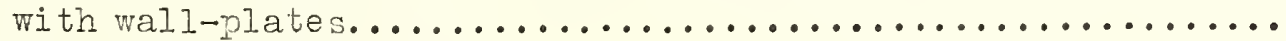
Watchmen's-Report-Apparatus.

$W-0-821 a$ $*$ W $-1-131$

$\pi-P-146$

TiT- -36

$m-R-41$

$* W-R-151$

$* w-S-871$

*w-S-893

$* \operatorname{Tin}-\mathrm{S}-896$

$\pi-7-101$

\section{FURNI URE}

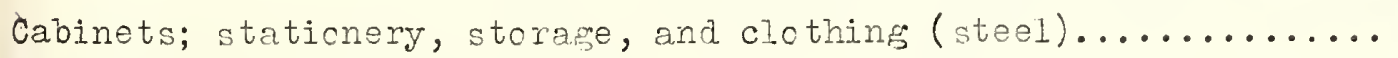

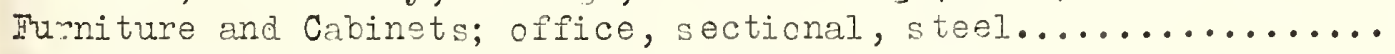

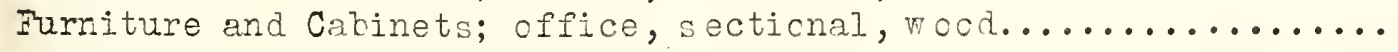
Locliers; clothes, steel..

*AA-C- 31

*AA- $\mathrm{F}-791 \mathrm{~B}(10 \notin)$

$* A A-F-801$

Shelving; steel, stcrage.

*AA-I-486

*AA-S-271

\section{GIASS AND GIASSWARE}

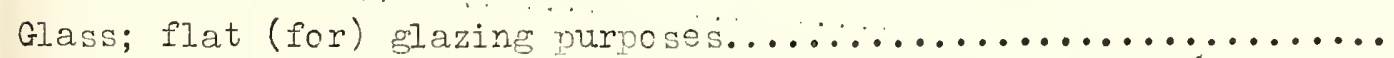
Glass; flat, glazing (for) transmitting not less than $25 \%$ of ultra-violet radiation at wave length 302 milimicrons....

*DD-G 451 $D D-G-476$ 


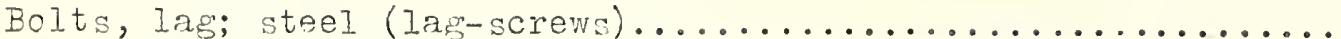
Bolts; Nuts; Studs; and Pap-Rivets, (and material for same).....

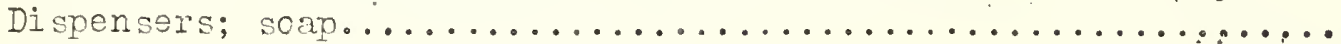

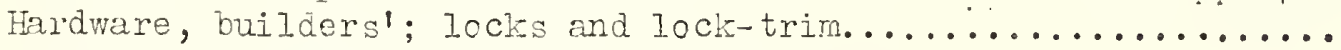

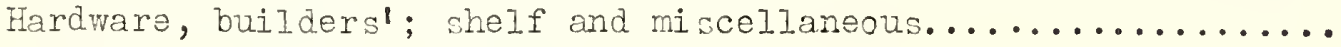

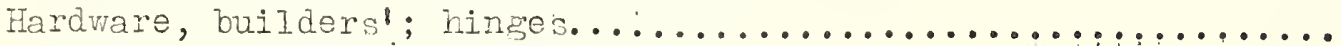

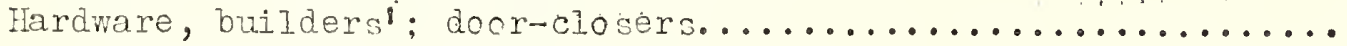
Hardware and Fittings; (for) lavatory partiticns and

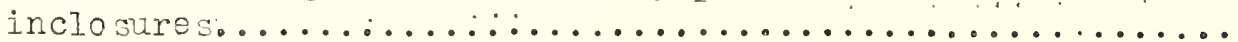

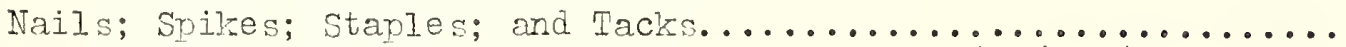

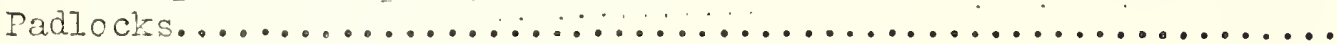
Eins; cotter, split.

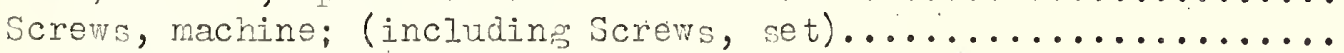

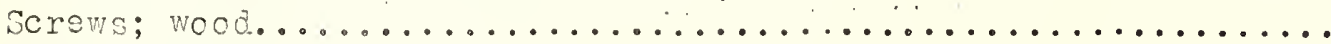
Tharnbuckles. Wool; steel.

\section{INSUIATING MAIPRIAIS}

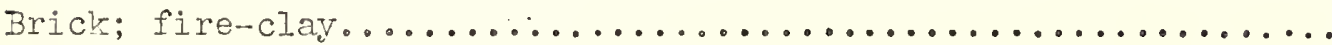

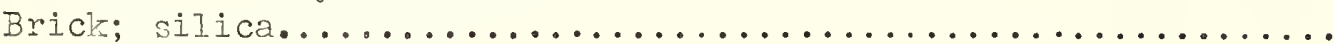

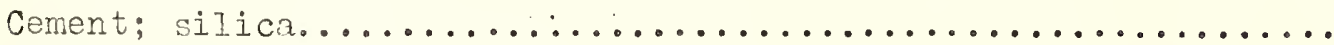
Clay; fire.

Compcund; plumbine-fixtire-setting.

Cork; compressed (corkboard):

Cork; granulated, insulating.

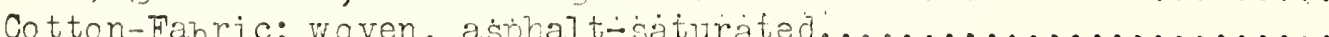

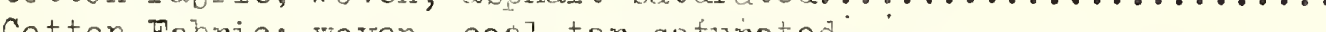

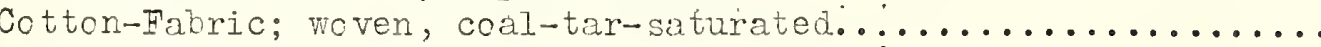
Felt; asphalt-saturated (for) flashings, roofing, and

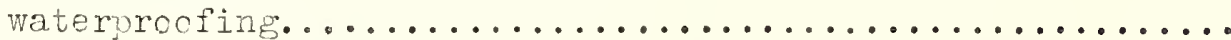

Felt; coal-tar-saturated (for) rocing and waterprocfing....... Filler, Expansion-Joint; Freformed; nonextmaing and resiliert-

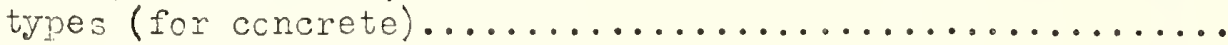

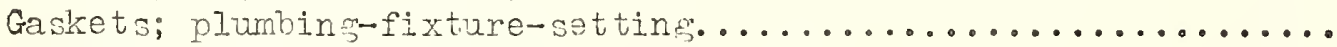

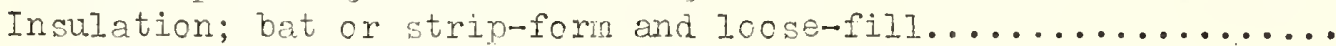
Insulation (vegetable or wood-fiber); blanket, felt,

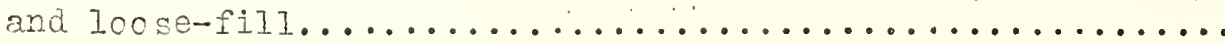

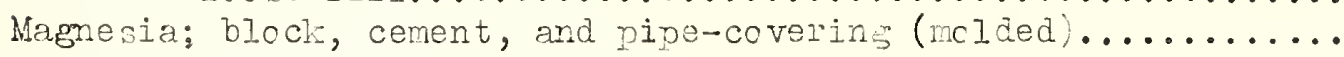

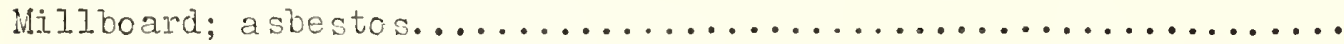
Mineral-Wool, impregnated; blanket, block, and pipe-covering

(molded), (for low temperatures).

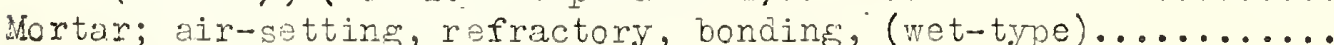

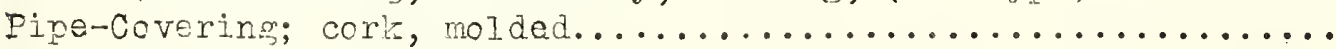

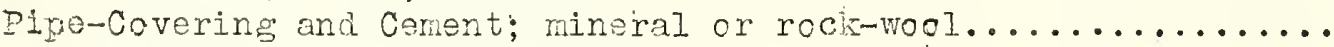

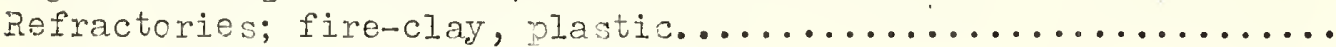

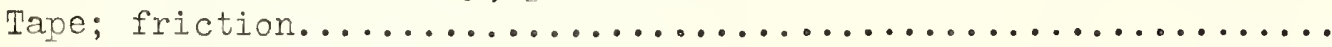

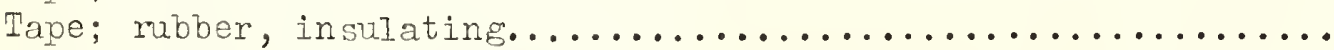
Tubing; flexible, nonmetaliic.

\section{KNIT GCCDS, NETIING, AND WEBBING}

$* F F-B-561$

* FE-B-571a

*FA-D-396a

*FE-H-1C6

$* F F-H-1 I I$ ( $10 \phi)$

*F-i-116a( 10

* FT-H-I2la

*FF-II-136

* ET-N-101

* FI-P-IOIa

* $\mathrm{E}-\mathrm{A}-\mathrm{P}-386$

$* \mathrm{~F} T-\mathrm{S}-91$

* FT-S-111

* 9 T-1 $-7-792$

* FT- T-5 556

*-7II-B-671b

* HI-B-68I

HHE-C- 176

* Hi-C-45I a

IHI-C -536

*HH-C-56]a

* III-C-571a

*HII-C-581a

HII-C-59I

* HII-F-191

*HI-F-201

$\mathrm{HIH}-\mathrm{F}-34 \mathrm{I}$

$H H-G-116$

IEI-I-521b

FII-I- 571

* $211-3-61$

*

IFH $-M-371$

$H H-M-611$

$\mathrm{H}-\mathrm{P}-38 \mathrm{I}$

IEH-P-386a

* EH-R-191

* IE-T-IOIa

* HiH-T-IIla

* HEl-T-79I 
Iumber and Timber;

Lumber and Timber; hardwood.

softwood.
*MM-I-736

*MN-I-751a

\section{MACHINERY}

Air-Conditioning-Units (room-coolers); electric-motor-driven, portable.

$00-A-361$

\section{METAIS}

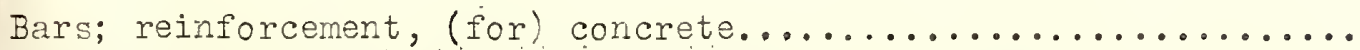

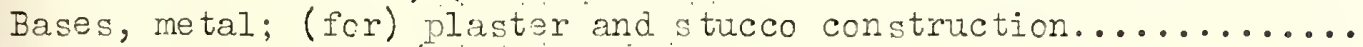

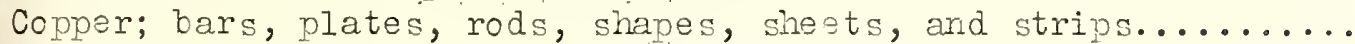

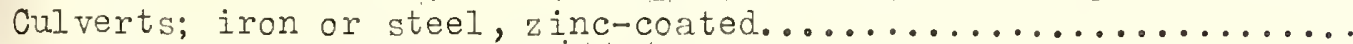
Iron and Steel; sheet, black and zinc-coated (galvanized).......

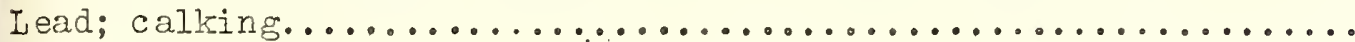
Iead; sheet.

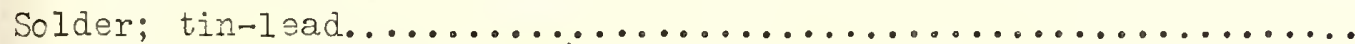
Steel; stay-bolt (boiler).

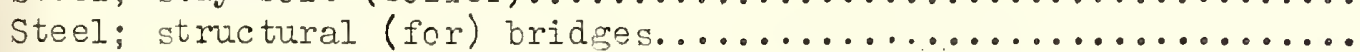

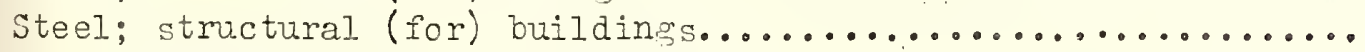
Steel, Corrosion-Resisting; plates, sheets, strips, and

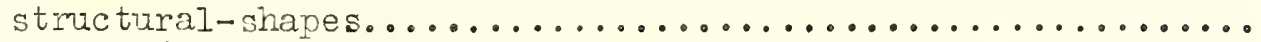
Terne-Plate (longuternes). Terne-plate (roofing-tin).

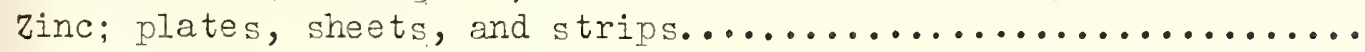

\section{METAI PRODUCTS}

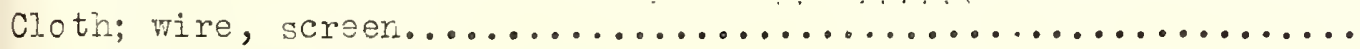

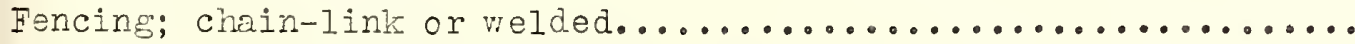
Fencing; wire (barbed, netting, and woven), black and

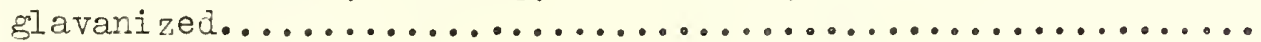

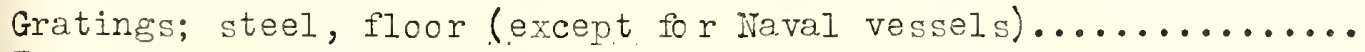

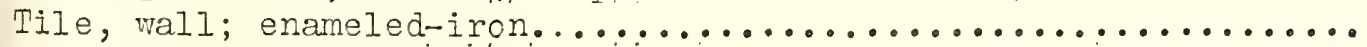
Treads; safety, netallic..

\section{MINERAIS AND PRODUCTS (nonmetallic)}

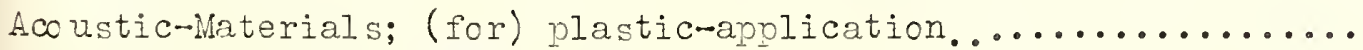

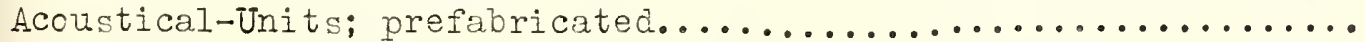

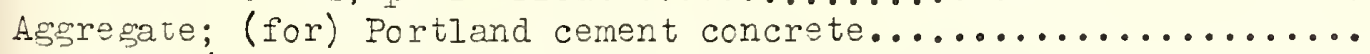
Asphalt; (for) built-up roofing, waterproofing and

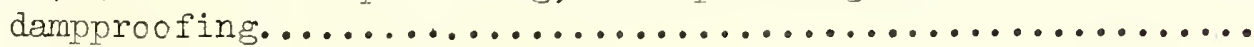

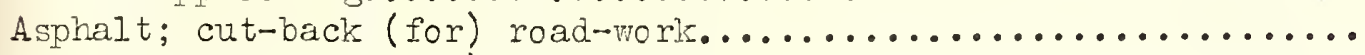

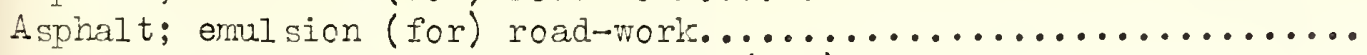
Asphalt; petroleum, type PAF-1-25, (for) joint filler

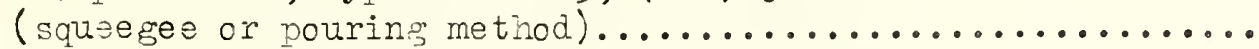

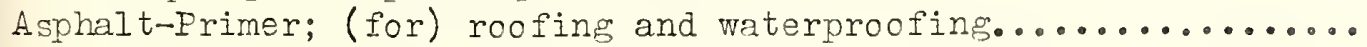
Asphalt; (for use in) road and pavement constmetion............

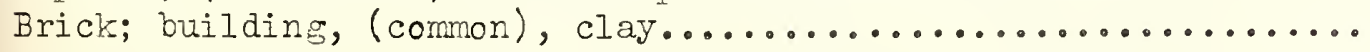
Brick; concrete..................................

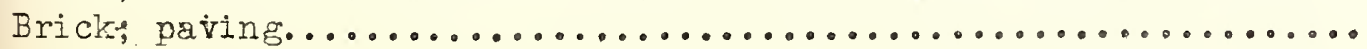

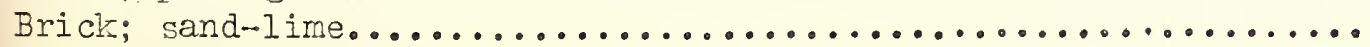

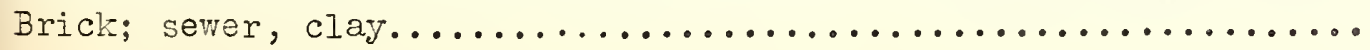

*RR-C-451a $* R R-F-191$

$* R R-F-221$

$* \mathrm{RR}-\mathrm{i}-66 \mathrm{Ia}$ RR-T-421

*RR-T-661

$S \tilde{S}-\mathrm{A}-111$

$\mathrm{SS}-\mathrm{A}-118$

$S S-A-281$

* SS-A-666

SS-A-671

- SS-A-674

* SS-A-696

* SS-A-701

* SS-A-706

* SS-B-656

* SS-B-663

* SS-B-671a

* SS-B-681

* SS-B-691 


\section{MINERAI AND PRODUCTS (nonmetallic) (Cont.)}

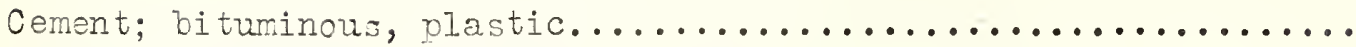

Cements, hydraulic; general specifications (me.thods for

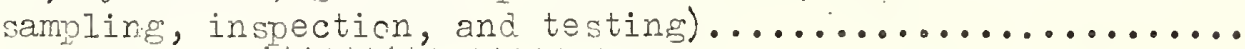

Cement; Keene's.

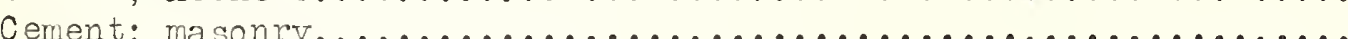

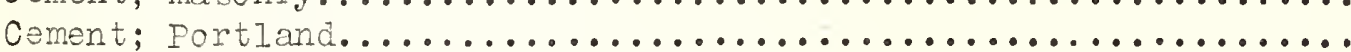

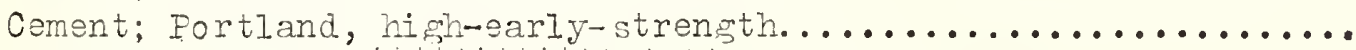

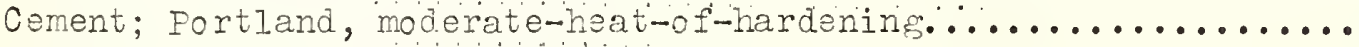

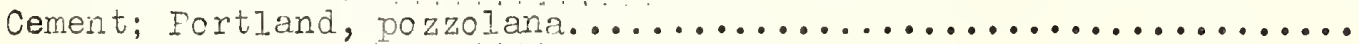

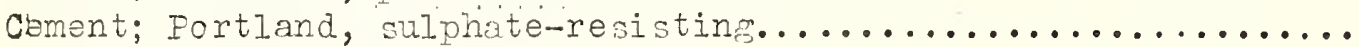

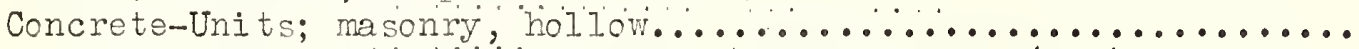

Crushed-Stone, Crushed Gravel, and Crushed-Slag; (for)

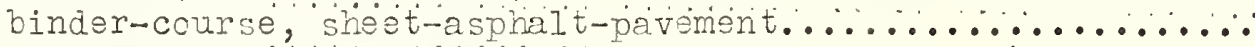

Crushed-Stone, Crushed-Gravel, and Crushed-Slas; (for)

bituminous-concreté-base or sưrace-course. $\ldots \ldots \ldots \ldots \ldots \ldots \ldots \ldots$

Crushed-Stone and Crushed-siak; (for) "bituminous-madada-

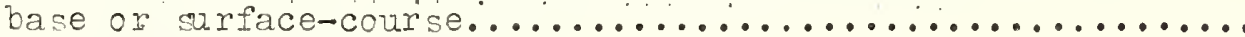

Crushed-stone and Crished-silag and Gravel; (for)

bituminous surface-treatment......................

Crushed-Stone and Crusined-Slag; (for) waterbound-base

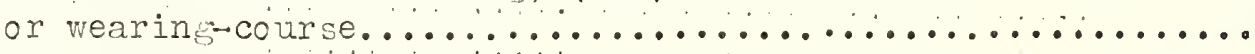

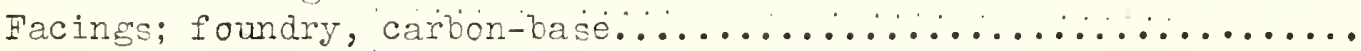

Granite-Blocks; recut granite, and durax sranite pavements......

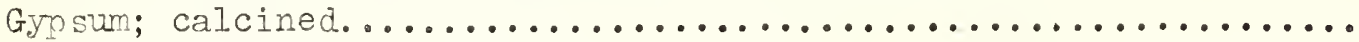

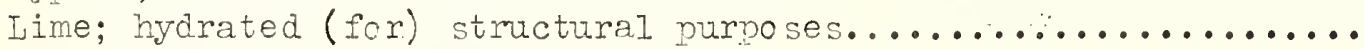

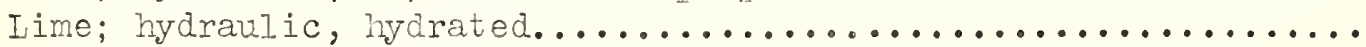

Materials; (for) cushion course, brick, stone-block, or wood-

block pavements (sana, slas," limestone; 'screenings, etc.)... Mineral-Filier; (for) sheet asphalt or asphaltic concrete

pavements (Portland cement, ilmestone dust and dolomite

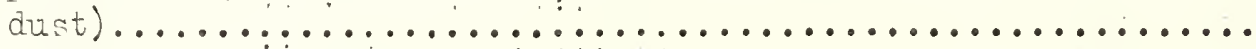

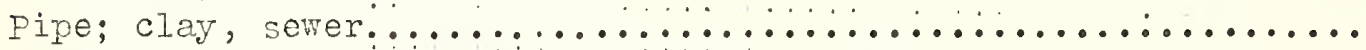

Fipe; concrete, non-pressure, non-reinforced and reinfóced.....

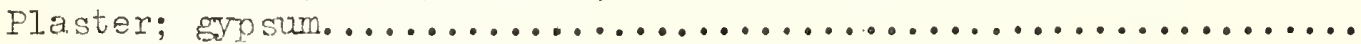

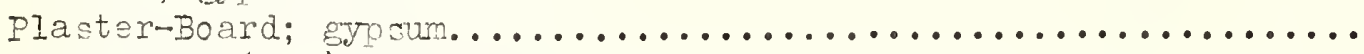

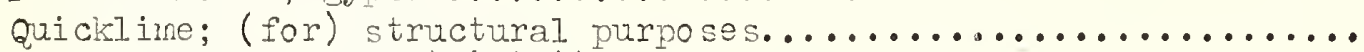

Road and Paving-Materials; general specifications (methods

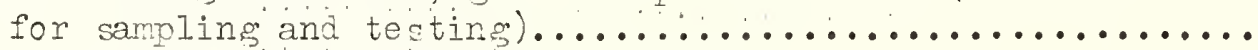

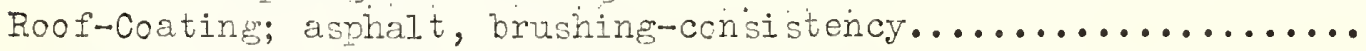

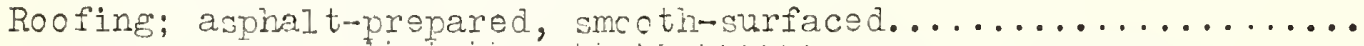

Roofing; asphalt and asbestos-prepared, mineral-surfaced........

Roofing and Shingles; a sphalt, prepared', mineral-surfaced.......

*SS-C-15,3

$S S-C-158(10 \phi)$

* SS-C-161

* SS-C-181.

* SS-C-191a

$S S-C-201$

SS-C-206

$\mathrm{SS}-\mathrm{C}-208$

SS-C-211

* SS-C-62I

$S S-C-726 a$

SS-C-73ia

$S S-C-736 a$

$S S-C-741 a$

$S S-C-746 a$

* SS-F-111

SS-G-651.

* SS-G-901

* SS-I-351

SS-I-361

$S S-M-51$

$S S-M-35 I$

$S S-P-361$

* $S S-F-371$

* SS-P- 401

* SS-P-431a

* SS-Q- 351

SS-R-406(106)

* SS-R-451

* SS-R-50I

* SS-R-5II

* SS-R- 521 
Sand; (for) cement-rnortar-bed, (for) bricl, stcne-block or

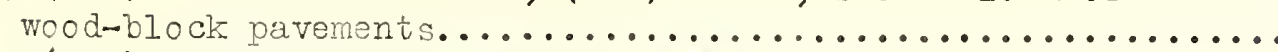

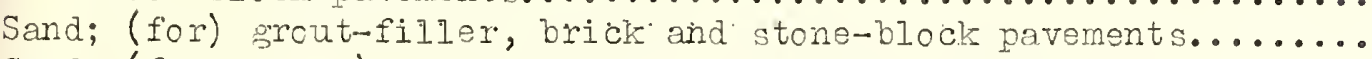
Sand; (for use in) sheet asphalt or bituninous concrete

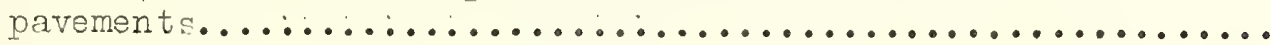

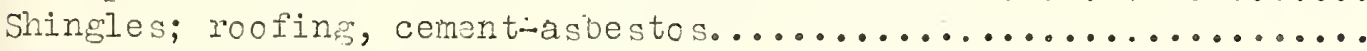

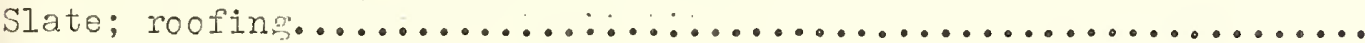

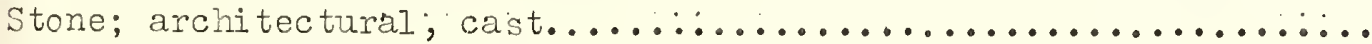
Tile; asphalt..............

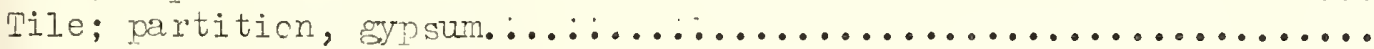

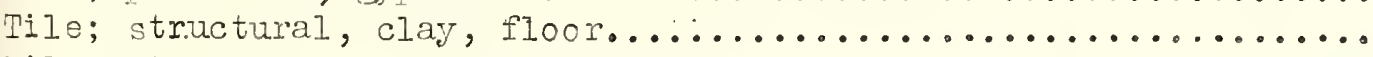

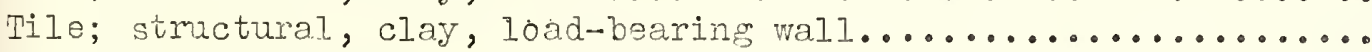

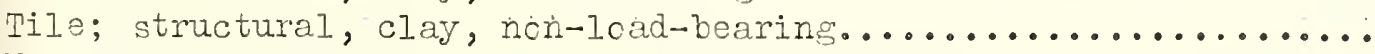
Wall-Board; gyo sum. .

\section{PAINTS, PIGMUITS, VARNISHES AIND PECDUCTS}

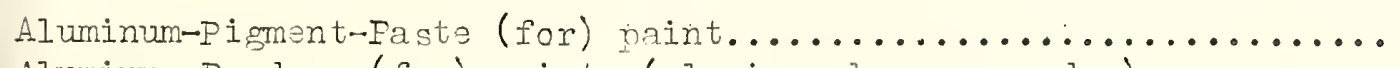

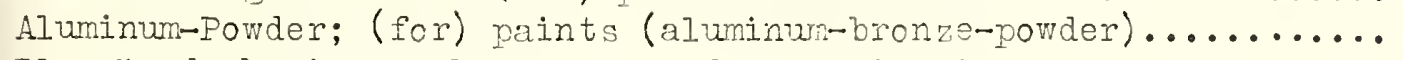

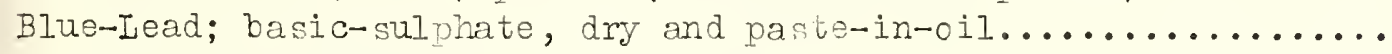

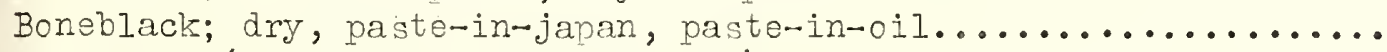

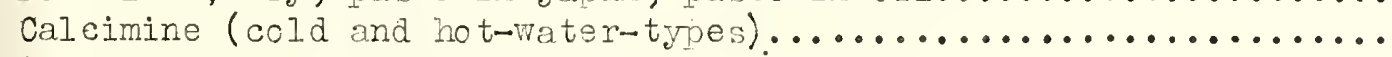
Chrome, green; oxide..

Chrome, green; pure, dry, paste-in-japan, paste-in-oil. Chrome, yellow; äry, paste-in-japan, paste-in-oil

(lemon, mediun, orange).

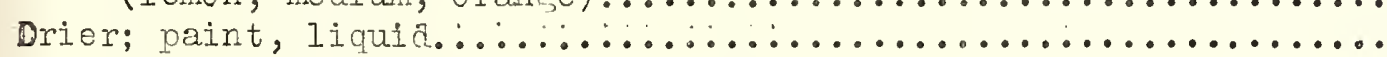

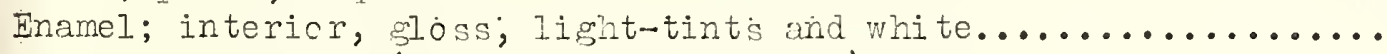

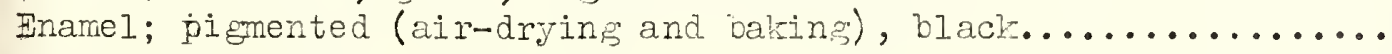

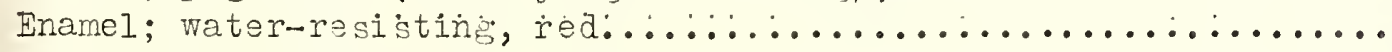

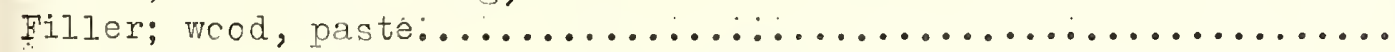

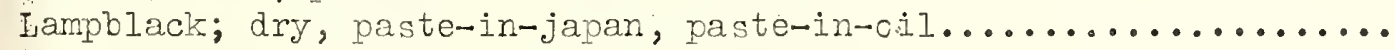

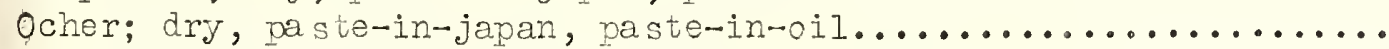
Paint, Blue-Iead-Base; basic sulphate, Linseed-oil, ready-nixed...

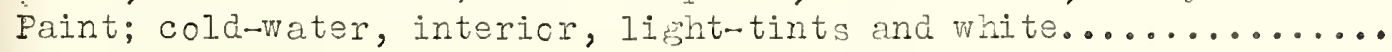

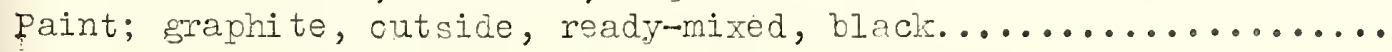
Paints; iron-hydroxide and ircn-cxide, ready-inixed and

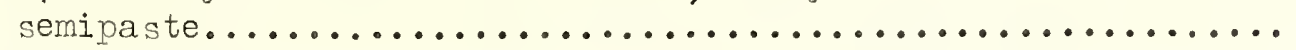
Paints; lead-zinc base, ready-mixed and semipaste, white

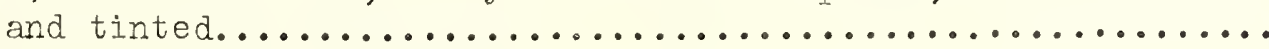
Paints; oil, interior, essshell-flat-finish, ready-mixed

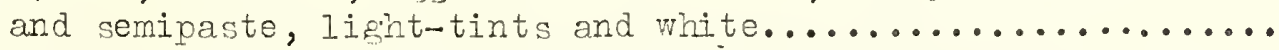

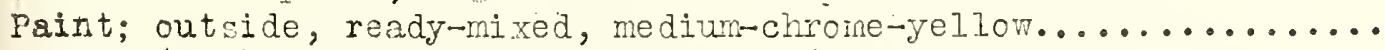
Paint; (for) prining plàster-surfaces (plaster primer and sealer).

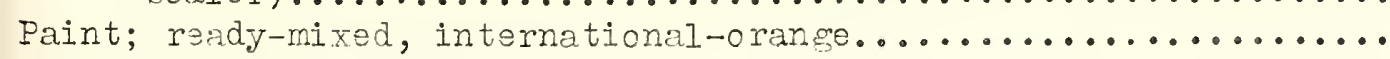

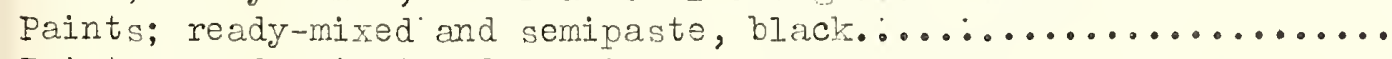

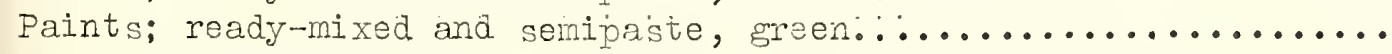

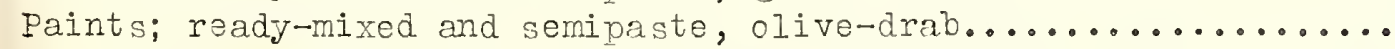

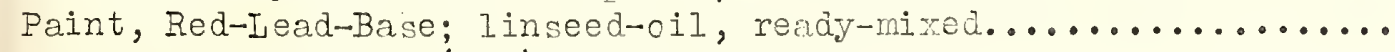

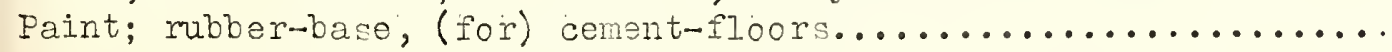
Paints; titanium-zinc, and titanium-zinc-lead, outside, ready-mixed, white.

$T I-A-466$ $* \operatorname{tr} T-A-476$ $*$ * $1-3-486$ $* T+B-601$ $T r-C-96$ *TT-C-231. *TT-C-236

* *TT-D-651 * Tr-2-506a * TI-E-521 *Tr-E-531a *IT-E-336 *IT-I-7I * $19-0-111$ $T T-P-20$ $* T T-P-23 a$ $T T-P-27$

$* \mathrm{TP}-\mathrm{P}-3 \mathrm{I}$

*Tr-P-36a

*[TI-E-5Ia *TT-P-53

*

$T-P-59$

*TI-P-61

*TT-P-71

* TT-P-81

IT-P-86

TT-P-91

*IT-E-10la 
Primer; paint, rinc dust-zinc cxide (for galvanized

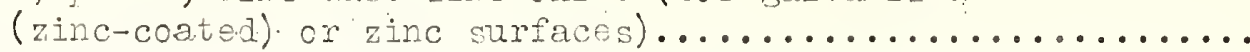

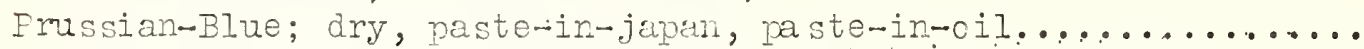
Futty; pure linseed cil, (fcr) wood sash-slazing..............

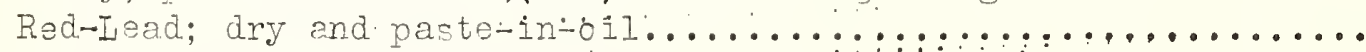

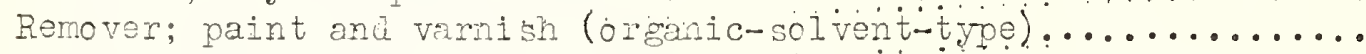

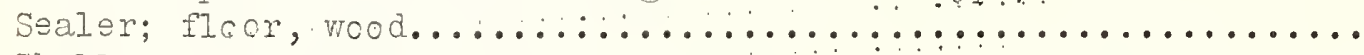

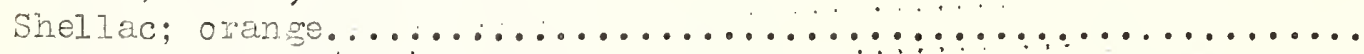

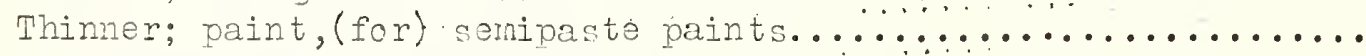

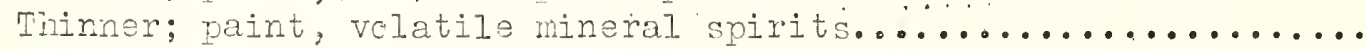
Ultramarine Blue; dry, paste-in-japan, paste-in-oil...........

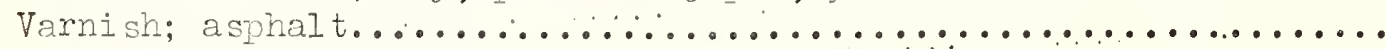

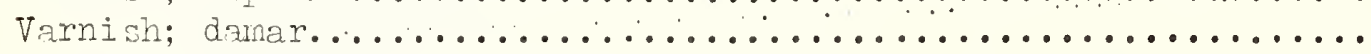

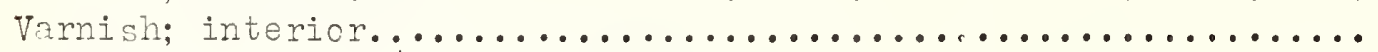

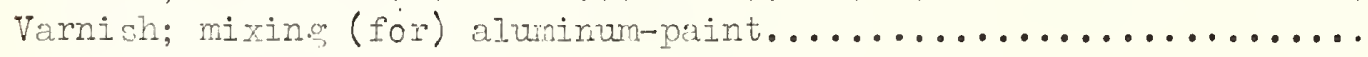

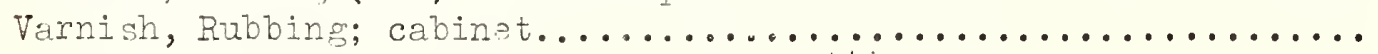

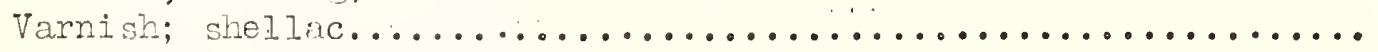

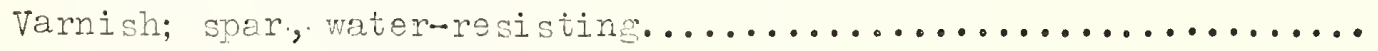
White-Iead; basic-carbonate, ary, paste-in-oil, and

semipaste containins volatile-thinner................ White-Iead; basic-silnhate; dry and paste-in-cil............. Tood-Preservative; ccal-tar creosote (fcr) ties and

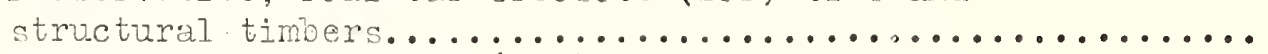
Wood-Freservative; creosote (ICr) brusin and spray-treatnent..... Wood-Freservative; creosote-coat-tar-sclution (for) ties .

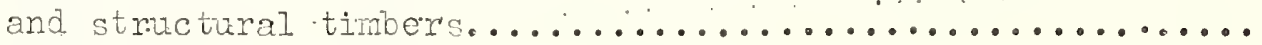

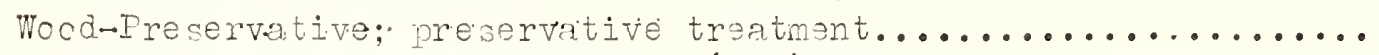
Wocd-Preservative; zinc-chloride (for)ties and

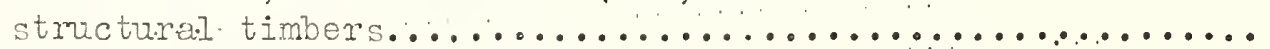

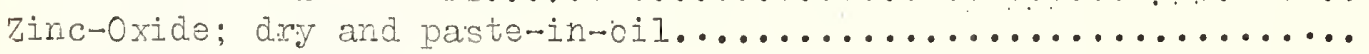

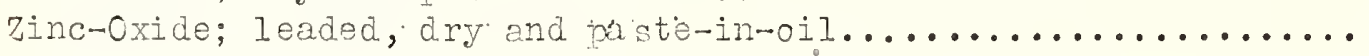
$T-P-641$ $* T-E-691$ $* T T-P-791 a$ $* T T-R-191 a$ $* T-R-251$ $T-5-176$ $* T-S-271$ $*+\mathrm{T}-\mathrm{T}-271$ $* T T-T-291$ $* \pi+-4-451$

*TT-V-5I $T T-V-61$ *TT- $-71 \mathrm{r} a$ * TT-V-8la $\mathrm{TT}-\mathrm{V}-86$ *TI- $\mathrm{T}-91 \mathrm{a}$ * TT-V-I2la

$* T-\pi-251 a$ *Tr-Ti-261a

$T T-\pi-556$ $T T-\cdots-561 a$

$T T-\pi-566$ TI-N-571a

$T T-\pi-576$ * ITI-Z-301 $* T T-z-321$

\section{PAPER AID PHCDITCTS}

Paper, Kraft; coneretemcuring; waterprcofod................ Paper; sheathing, watempof.........................

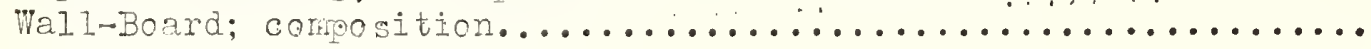
$U T-P-264$ *UTJ-P-536 *UU-iv-10la

\section{DETROIEIUI AMID ERODUUCTS}

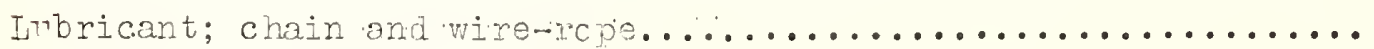

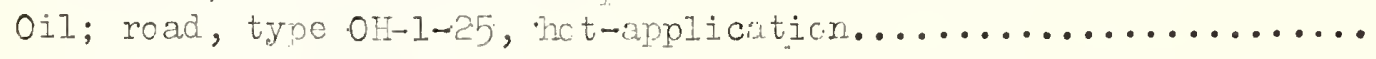

$* V V-I-751$ $* V V-0-751$

\section{EIPE, EIPE-TITIITGS, PLUNBIUG-FIXTURAS, TUBES, AND TUBIVG (metaIIIC)}

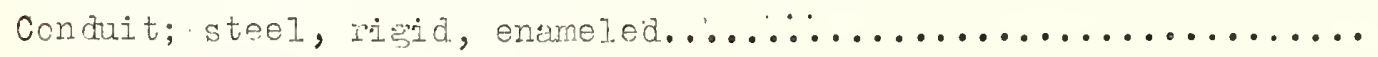

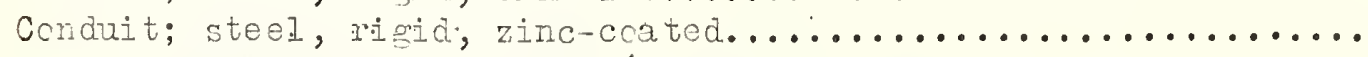
Flange-Dimensicns; standard; (classes 125 and 250 cast-iron

flanges;.c lasses 150 and 250 brcnue-planges) (for land use).

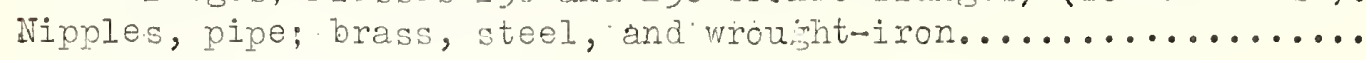

* TT-C-571 *wi-c-58Ia

WT-F- 406 *w-IT-351 


\section{PIPE, FIPE-EITIINGS, PLUBBING-IIXUUPS, IUBFS, AIVD TUBING}

(metallic) (Crnt.).

Pipe; brass, seamless, irçn-pipe-size, standard and

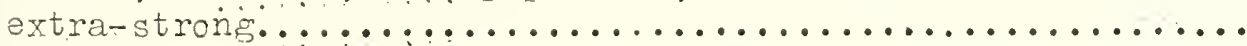

Fipe, cast-iron; drainage, vent, and waste, (threaded).........

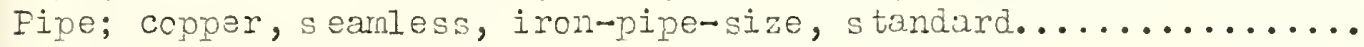

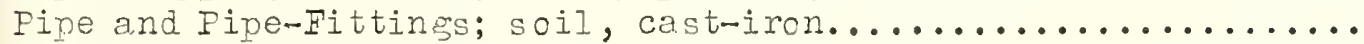

Pipe; steel and ferrous-alloy, wrought, iron-pipo-sire.........

Pipe; water, cast-iron (bell and spisot, and bolted-joint)......

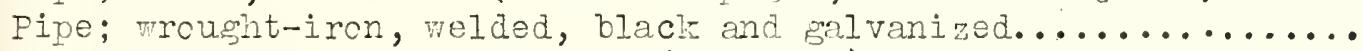

Fipe-Fittings; brass or bronze (threaded), 125-pounds..........

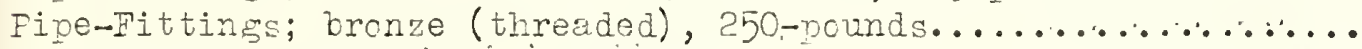

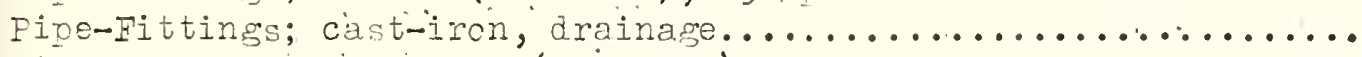

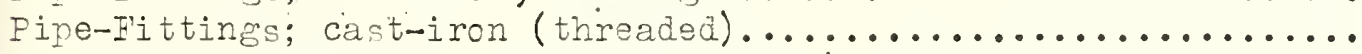

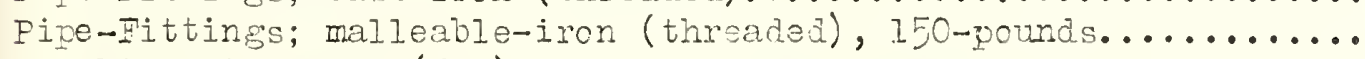

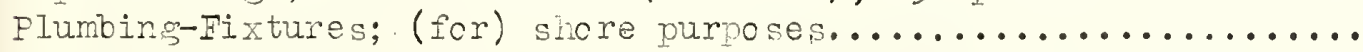

Traps; radiatcr, themostatic, brass or brenze, low-pressure,

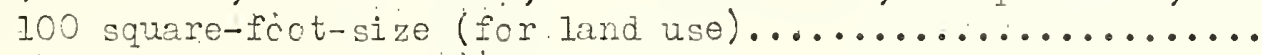

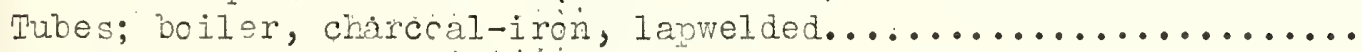

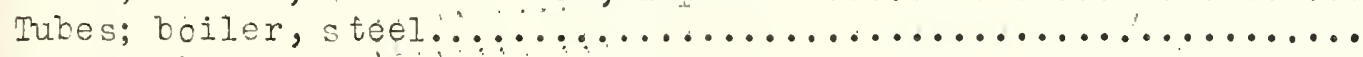

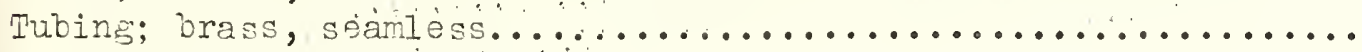

Tubing; copper, seariless (for general use with I.P.S.

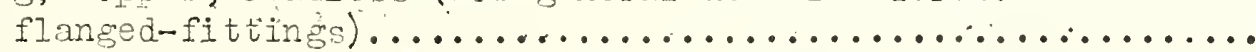

Tubing; copper, scamless (for use with soliered or flared-

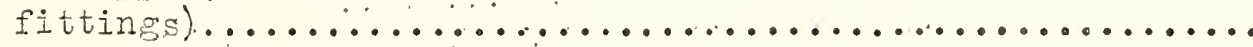

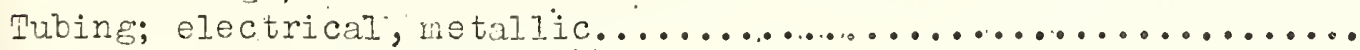

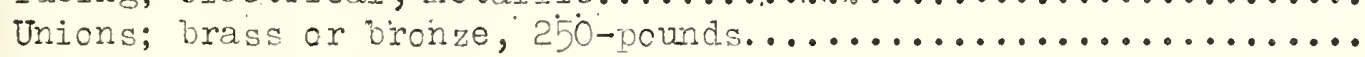

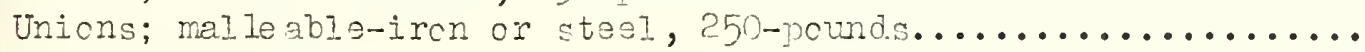

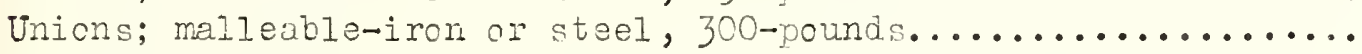

Valves, brass or bronze; angle and slcbe, 150-pounds, threaded

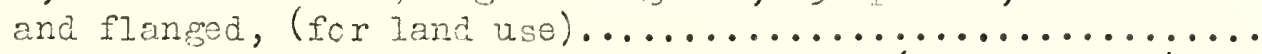

Valves, gate; 125 -pound, threaded and 1 lanped, (for 1 and use)....

Valves, radiator; air, themostatic (pravity-stemheating-

systems).

$* W W-P-351$
$W W-P-356$
$* W-P-377$
$* W T-E-401$
$* W-P-403 a$
$* W i-P-421$
$* W-P-441 a$
$* W W-P-448(10 \phi)$
$* W-P-461$
$W W-P-491$
$* W W-P-501 a$
$* W-P-521 a$
$* W W-P-541(10 \phi)$

$* \pi T-T-696$

Wi- -721

$* \pi+T-731 a$

*⿻一巛w -191

$* w-T-797$

* mir-T-799

*wir-T-806a

*TW-U-516

* *Tr-J-531

$* W T-\mathrm{U}-536$

$W \because-V-51$

$w+V-76 b$

WW-V-151

\section{RUBBER AIND ILUBDER-GOCDS}

Cushicn, underlay; carpst and ma, sponge-mbber..............

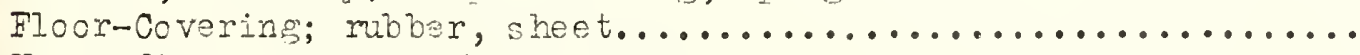

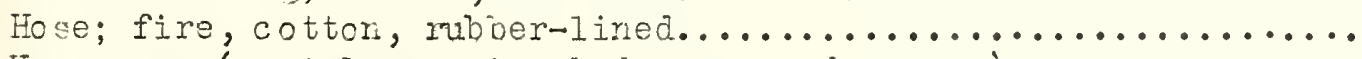

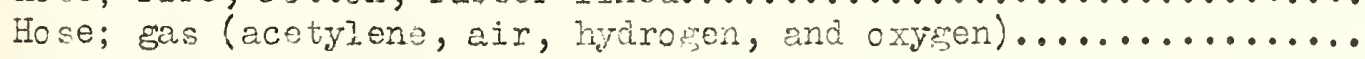

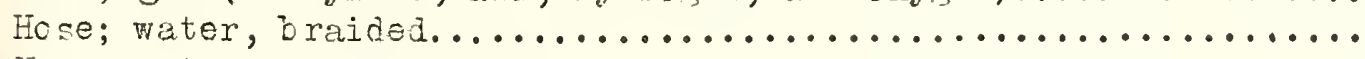
Hose; water, wrapned.

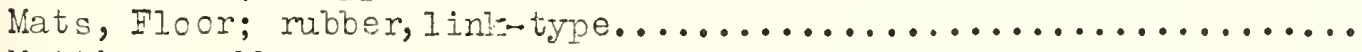

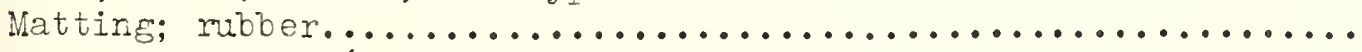
Matting; rubber (for use a round electrical apparatus or circuits not exceeding 3,000 volts to grcund)............

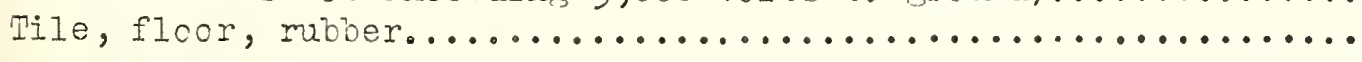

$\mathrm{ZZ}-\mathrm{C}-8 \mathrm{ZI}$

$* z z-\pi-461$

$* 27-4-451$

*22-I-461a

* ZZ-E- 601

*ZZ-H-6II

$27-x-46$

* $2 Z-11-71$

* $72-1-81$

$2 Z-1-301$ 


\section{TEXTIES (yardage)}

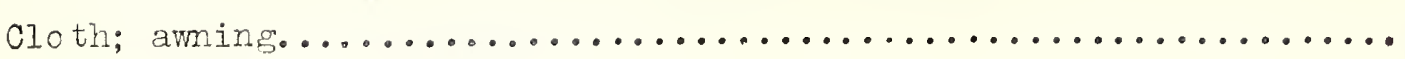

Cloth; shade.

\section{TEXTIIE PRODUCIS}

Shades, windcw; rollers, slats, cords, and accessories........... DDD-S-251

\section{VEGZTABIE PRODUCTS}

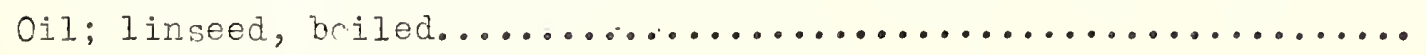

Cil; Iinseed, raw...

$* J J J-0-331$

*JJJ $-0-336$

\section{WOOD ERODUCTS}

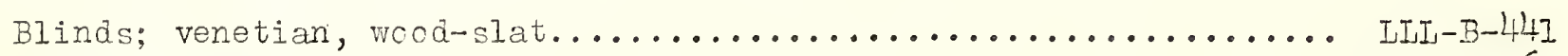

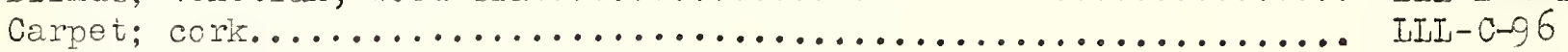

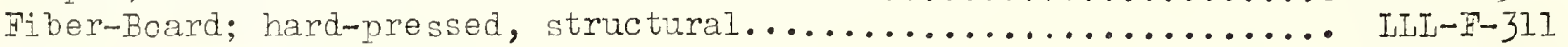

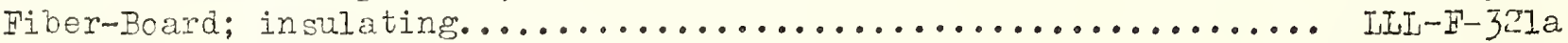

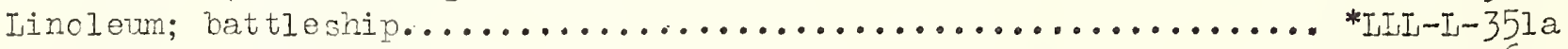

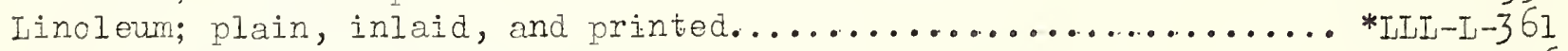

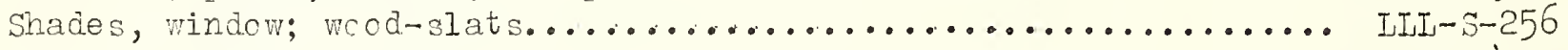

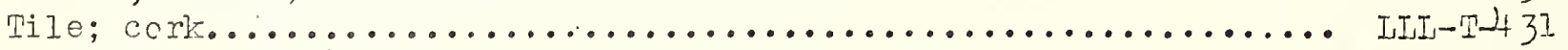

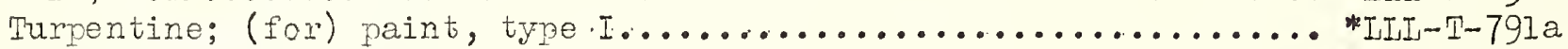

Turpentine; (for) paint, type II..........................III-T-792 
Recommended Minimum Requirenent for Mascnm Tall

Construction (and Mcdifications theretc).(1924).......BH 6

Minimum Iive Loads Allowable for Use in Design of

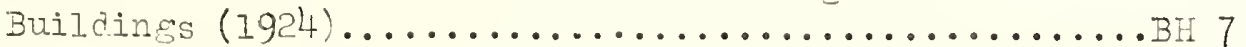

Recomnended Jinimu Requirements icr Plumbing (1932)........BH 13

Recomended Ninimum Regirenents for Fire

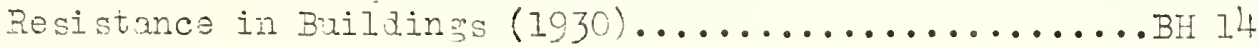

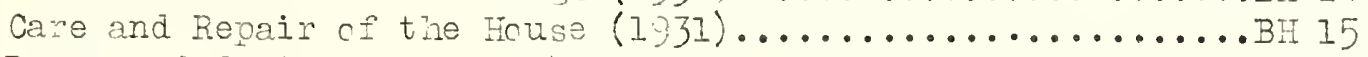

Reccmended Minimum Reguirements for Small Drelling

Construction (1932) ............................. 18

$15 \phi$

$10 \phi$

$50 \phi$

$10 \notin$

$15 \phi$

$I 0 \varnothing$

Preparation and Revision of Building Codes (1939)..........BMS 19

Flumbing Manual: Report of Subcomittee on Plumbing

$15 \phi$

of the Central Housing Committee cn Research,Design,

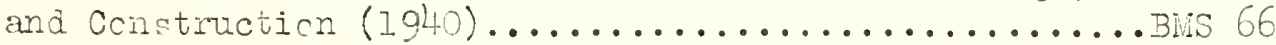

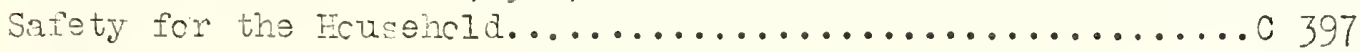

Design and Constructicn of Buildine Exits (1935)............ 151

Publications Relating to Accident Ereventicn

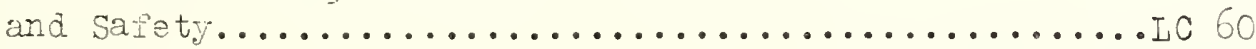

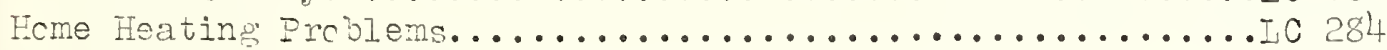

Free

I!

List of Published Material Relating tc Inme

Building and laintenance $(1938) \ldots \ldots \ldots \ldots \ldots \ldots$. IC 287

House Plan Services........................... 428

Building Materials, Building Standards, Fome Building:

Fublications of the Naticnal Bureau cf Standards.......IC 592

List of Publications of Interest tc the General Public (1940)IC 586 Publicaticns Relatine to Brilding Coà es and

Construction Iractice, Home Building--Building

Naterial Specificaticns-. Fome Maintenarce (1040).......IC 599

Building Materials and Structures Reports (Iist) (1940).....IC 552

Building Regratation: Fublications issued br the

Naticnal Bureau of Standards (Descriptive Iist) (1939). IC 555

Technical Information cn Building Materials (Iist) (I.938)... ....

Services of the National. Bireau co Standards to the

Home Building Industry and to the Househola................

The Certification Plan, Its Significance, Scope and.

Application to Selected Pederal Specificaticns and

Connercial Standards.......................... 607

11

Publications which are marked "Froe" nay be cbtained frcm the Divisicn of Cojes and Specificaticns, Naticnal Bureau cf Standards, Washington, D. C. 
Detach and mill to

Superintendent of Dccuments,

Government Printine; Office,

Date

Washinton, D. C.

Enclcsed is \$_... (ncney order, New Ycrk draft, cr coin) fer the following vublicaticns:

Series

Publication

Price
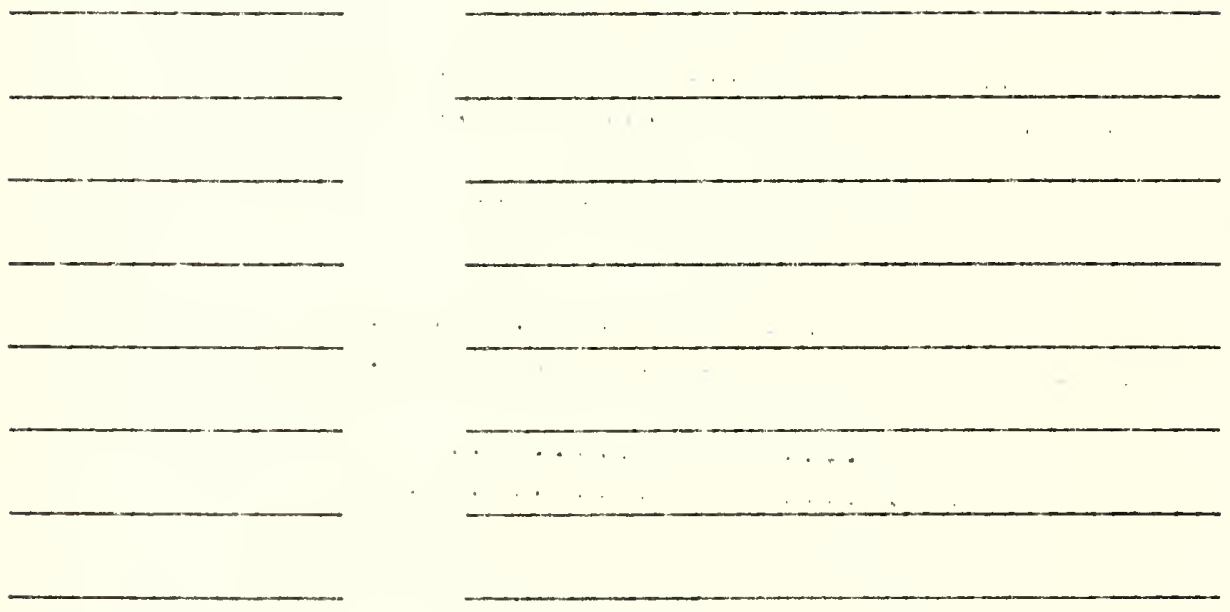

ICTAI
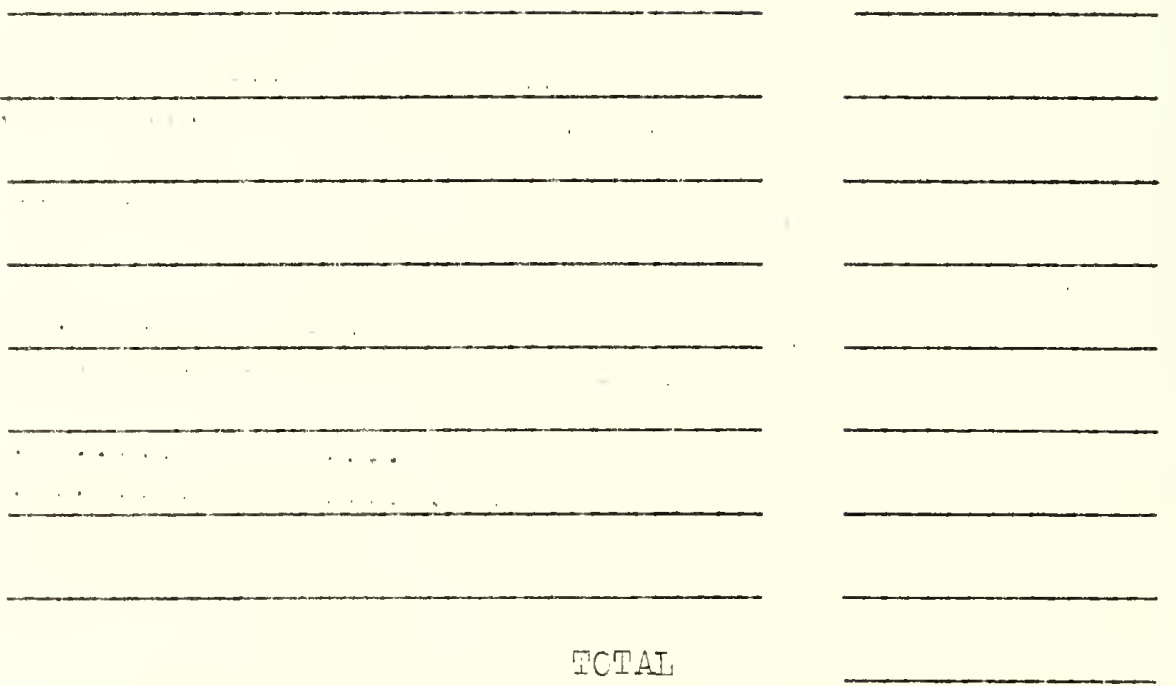

Niame:

Adaress: No. Street:

City: State:

$-14-$ 